WIENER SLAVISTISCHES JAHRBUCH, Band 57/2011, 215-218

(C) 2011 by Österreichische Akademie der Wissenschaften, Wien

S TEFAN S IMONEK

\title{
Wiener Notiz zu Aleksandar Flaker (1924-2010)
}

Im Herbst vergangenen Jahres erreichte das Institut für Slawistik der Universität Wien die traurige Nachricht, dass der prominente kroatische Literaturwissenschaftler Aleksandar Flaker am 25. Oktober 2010 im Alter von 87 Jahren in Zagreb verstorben ist. Flaker wurde 1924 im polnischen Białystok geboren, er studierte Slawistik und Philosophie an der Universität Zagreb, 1954 erfolgte die Promotion, 1957 dann die Habilitation. 1965 wurde er zum Professor für russische Literatur an die Universität Zagreb berufen, wo er auch über seine Emeritierung im Jahr 1989 hinaus bis zum Jahr 2002 lehrte; daneben war Flaker seit 1991 auch ordentliches Mitglied der Kroatischen Akademie der Wissenschaften und Künste sowie Mitglied mehrerer anderer zentraleuropäischer Akademien außerhalb von Kroatien. ${ }^{1}$ Als wichtige Arbeitsgebiete Flakers kristallisierten sich im Lauf seiner wissenschaftlichen Karriere die russische und die kroatische Avantgarde, die Literaturtheorie (erinnert sei in diesem Kontext an die 1976 in Form einer Monographie vorgelegte, immer noch relevante Konzeption der Stilformation) und - besonders in den späteren Jahren - die Intermedialität von Literatur und bildender Kunst und die slawischen Literaturen nach dem Zweiten Weltkrieg heraus; letzteren widmete Flaker seine auch in deutscher Sprache veröffentlichte Monographie Modelle der Jeans Prosa (Kronberg/ Taunus 1975). Dabei firmierte das elaborierte Nachzeichnen sowohl des allgemein slawischen als auch des darüber hinaus reichenden europäischen literarischen und

${ }^{1}$ Vgl. in Auswahl folgende rezentere Positionen zum Werk Flakers: J. Užarević (Hg.), Aleksandar Flaker, professor emeritus Facultatis Philosophicae Universitatis Studiorum Zagrabiensis, Zagreb 2002; J. Užarević (Hg.), Oko književnosti: osamdeset godina Aleksandra Flakera, Zagreb 2004; dort ein Schriftenverzeichnis Flakers auf S. 351-383; Russian Literature LVI (2004), 1-3 (Festschrift für Aleksandar Flaker); über Internet einsehbar sind zwei Nachrufe auf Flaker unter http://danas.net.hr/kultura/page/2010/10/25/0539006.html bzw. alternativ unter http://www.culturenet.hr/default.aspx?id=33866 sowie zusätzlich der Eintrag zu Flaker auf „Wikipedia“ unter http://de.wikipedia.org/wiki/ AleksandarFlaker (alle abgerufen am 22. 12. 2010). 
kulturellen Kontextes der jeweils behandelten Frage als grundlegendes Bindeglied zwischen all den zuvor erwähnten Arbeiten.

Für das Wiener Institut für Slawistik stellt das Ableben Aleksandar Flakers insofern einen besonders schmerzlichen Verlust dar, als die über Europa hinaus bis in die Vereinigten Staaten von Amerika ausstrahlende, intensive und breit gefächerte wissenschaftliche und pädagogische Tätigkeit des auch jenseits der Grenzen seiner Heimat anerkannten Slawisten und Komparatisten Flaker für einige Jahre auch eng mit dem Wiener Institut verbunden war. In der für das Institut ausgesprochen schwierigen und unerwartet lang andauernden Übergangsperiode gegen Ende der achtziger Jahre des vergangenen Jahrhunderts, die chronologisch in die Zeit nach dem krankheitsbedingten frühen Ende der das Institut prägenden Ära von Günther Wytrzens und vor die Berufung Sergej Averincevs auf den Wiener Lehrstuhl für ostslawische Literaturen fiel, zeigte sich Aleksandar Flaker mit dem Institut zutiefst verbunden, indem er seine im Wintersemester 1989/90 angetretene Gastprofessur in Wien mehrfach verlängerte und schließlich bis zum Wintersemester 1991/92 fortführte. Dabei deckte Flaker große Teile des Lehrbetriebs aus den Kernbereichen Russistik/Literaturwissenschaft sowie Vergleichende Slawische Literaturgeschichte ab (vgl. Anhang 1), hielt die gesamte Bandbreite an entsprechenden Lehrveranstaltungen (Seminare, Vorlesungen und Privatissima) und betreute mit großem Engagement eine für einen Gastprofessor beachtliche Anzahl literaturwissenschaftlicher Diplomarbeiten und Dissertationen, von denen einige späterhin auch in Buchform veröffentlicht wurden (vgl. Anhang 2).

Seine Verbundenheit mit dem Wiener Institut auch nach seiner Zeit in Wien selbst manifestierte sich weiter in zwei Aufsätzen, die im „Wiener Slavistischen Jahrbuch" veröffentlicht wurden: 1993 erschien in Band 39 der Zeitschrift Flakers Beitrag Proučavanje početaka moderne u hrvatskoj, srpskoj i slovenskoj književnosti (1970-1989) und im Jahre 1999 in Band 45 dann in Fortführung von Flakers intensiver und kontinuierlicher Auseinandersetzung mit dem Werk des kroatischen Autors Miroslav Krleža die Untersuchung Krležas Wiener Kaleidoskop, die ursprünglich auf einen Vortrag im Rahmen des Jubiläumssymposiums „150 Jahre Slawistik an der Universität Wien“ zurückgeht; auch auf Beiträge im „Wiener Slawistischen Almanach“, wie etwa Krležas maskierter Moskauer Text (Bd. 21/1988), wäre in diesem Zusammenhang zu verweisen. In dem herausragenden, von Flaker edierten und 1989 bei Droschl in Graz erschienenen Glossarium der russischen Avantgarde finden sich neben eigenen Aufsätzen des Herausgebers auch mehrere wichtige Beiträge von Aage A. Hansen-Löve und Rosemarie Ziegler, die über ihre akademische Ausbildung und ihre Lehrtätigkeit ebenfalls mit dem Institut in Wien verbunden waren. Der Darstellung Wiens allgemein in der kroatischen Literatur des 19. und des 20. Jahrhunderts sowie speziell im Werk des Slowenen Ivan Cankar schließlich sind Flakers Studien Das Stadtbild Wiens in der kroatischen Literatur (19. und 20. Jahrhundert) im Sammelband Wien als Magnet? Schriftsteller aus Ost-, Ostmittel- und 
Südosteuropa über die Stadt (Hg. G. Marinelli-König u. N. Pavlova, Wien 1996) sowie Cankarovi (bečki) prostorni kontrasti (Slavistična revija 45/1997) gewidmet.

Die in diesem Beitrag versammelten Materialien sollten nicht mehr beabsichtigen, als Aleksandar Flakers Wiener Jahre in deren wesentlichen universitären Parametern zu umreißen und Flakers Leistungen für das Institut für Slawistik der Universität Wien mindestens schlaglichtartig zu erhellen. Als einem der Wiener Schüler des großen kroatischen Gelehrten war es dem hier Bericht Erstattenden ein Anliegen, auf diese Dinge gebührend hinzuweisen und dem Verstorbenen seinen tiefen Dank auszusprechen.

\section{ANHANG 1:}

Verzeichnis der von Aleksandar Flaker am Institut für Slawistik der Universität Wien im Zeitraum von 1989 bis 1992 abgehaltenen Lehrveranstaltungen

Wintersemester $1989 / 90$ :

Das Theater bei den Slawen im 20. Jahrhundert (VO)

Die russische Prosa nach 1917 (VO)

Literaturwissenschaftliches Seminar: Russische Schriftsteller und die anderen Künste (SE)

Sommersemester 1990 :

Russische Literatur II (VO)

Das Theater bei den Slawen im 20. Jahrhundert (VO)

Literaturwissenschaftliches Seminar für Russisten (SE)

Seminar für Diplomanden (SE)

Wintersemester $1990 / 91$ :

Russische Literatur I (VO)

Die russische Avantgarde: Literatur und die anderen Künste (VO)

Literaturwissenschaftliches Seminar für Russisten (SE)

Seminar für Diplomanden (SE)

Sommersemester 1991 :

Der russische Roman (VO)

Die Avantgarde bei den Slawen (VO)

Literaturwissenschaftliches Seminar für Russisten (SE)

Seminar für Diplomanden (SE)

Wintersemester $1991 / 92$ :

Der russische Roman im 20. Jahrhundert (VO)

Die slawischen Literaturen im 20. Jahrhundert (VO)

Literaturwissenschaftliches Seminar für Russisten (SE)

Seminar für Diplomanden und Dissertanten (SE).

ANHANG 2:

Verzeichnis der von Aleksandar Flaker betreuten, an der Universität Wien approbierten Diplomarbeiten und Dissertationen im Zeitraum von 1991 bis 1996 (in chronologischer Reihenfolge)

Diplomarbeiten:

1. Jaremenko, Vladimir N.: Liričeskaja drama A. A. Bloka „Balagančik“: Komedija, tragedija, absurd? (1991) 
218

Stefan S i m o n e k

2. Bardell, Margaret: A. Platonov und der Ethnische Raum (1991)

3. Koller, Karin: Viktor Šklovskijs und Vsevolod Ivanovs experimenteller Trivialroman „IPRIT"(1992)

4. Rothpuller, Sabine: Symbolistische Prosa: Brjusov's „Ognennyj angel“ (1992)

5. Pohl, Sabine: Bulgakov und Science Fiction (1992)

6. Petković, Nevenka: Marina Cvetaeva und die Malerei (1993)

7. Adler, Birgit: Stalinismus in der russischen Prosa nach 1917 (1994)

8. Bracher, Christiane: Šostakovič und Gogol’: Oper und Erzählung „Die Nase“ (1994).

Dis sertationen:

1. Simonek, Stefan: Osip Mandel'štam und die ukrainischen Neoklassiker. Zur Wechselbeziehung von Kunst und Zeit (1991, Erstbetreuer - in Buchform gekürzt unter identem Titel München 1992)

2. Stepanov, Edith: Die Gestalt des Wissenschafters in der sowjetischen Literatur russischer Sprache (1991, Erstbetreuer)

3. Teichgräber, Stephan-Immanuel: Die Dekonstruktion der sozialistischen Mythologie in der Poetik Andrej Platonovs (1996, Erstbetreuer - in Buchform unter identem Titel Frankfurt am Main 1999).

10.08 .92

Shr jeehsher Herr Dohhor Lampe,

beiliegend ist meine Vuressdrift ander"Empfangs-

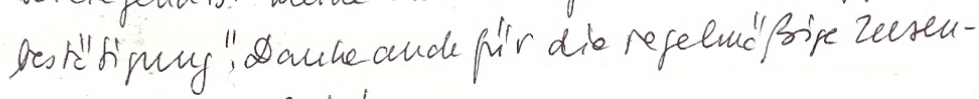
dungmeiner Rost!

Huen, Herer Fran Genonlin und allen in hustilut w'ihrend dieser tilze duwesenden mins dhe ich für den Rest der Ferien die besté Ekvoung.

Wirdes ande in Merbst eimige Pnifingengeben?

Ther

A. Telear 